\title{
RETROGRADE JEJUNO-GASTRIC INTUSSUSEPTION: A RARE CAUSE OF INTESTINAL OBSTRUCTION AFTER GASTROJEJUNOSTOMY
}

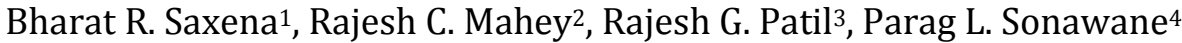

\section{HOW TO CITE THIS ARTICLE:}

Bharat R. Saxena, Rajesh C. Mahey, Rajesh G. Patil, Parag L. Sonawane. "Retrograde Jejuno-Gastric Intussusection: A Rare Cause of Intestinal Obstruction after Gastrojejunostomy". Journal of Evolution of Medical and Dental Sciences 2014; Vol. 3, Issue 22, June 02; Page: 6200-6203, DOI: 10.14260/jemds/2014/2722

\begin{abstract}
Gastrojejunostomy is a commonly performed bypass procedure for various intraabdominal pathologies. Jejunogastric intussusception is a rare and serious complication following gastrojejunostomy. It was first described in 1914 by Bozzi. These patients may present with features of acute onset intestinal obstruction many years after the primary surgical procedure. Such patients present with a classical triad of sudden onset epigastric pain, vomiting with or with-out hematemesis and a palpable epigastric mass. Upper G.I endoscopy is certainly diagnostic and computed tomography of the abdomen could also help in diagnosis. There is no medical treatment for acute jejunogastric intussusception and the correct treatment is surgical intervention as soon as possible. A mortality rate of $50 \%$ has been reported if operated after $48 \mathrm{hrs}$ of intussusception. The rarity of the complication and the need for urgent surgical management have prompted us to report this case of a 55years old patient having retrograde intussusception, 30 years after the gastro-enteric anastamosis.
\end{abstract} KEYWORDS: Gastrojejunostomy, Intussusception, Intestinal obstruction, Gangrene.

INTRODUCTON: Retrograde intussusception is a rare and lethal complication of gastrojejunostomy. It was first described in 1914 by Bozzi.[1,3,4] Around 200 cases have been reported in English literature till now. ${ }^{[2,3]}$ This may happen many years after the primary surgical procedure. These cases present with cardinal features of intestinal obstruction, pyrexia, features of dehydration and rarely hematemesis. There may be severe compromise of bowel vascularity progressing to gangrene which requires urgent surgical intervention. A mortality rate of $10 \%$ has been reported if the operation was performed within 48 hours and about $50 \%$ thereafter.[4,5]

CASE PRESENTATION: A 55 year old male was referred to our centre with primary complaints of pain in abdomen since 8 days, bilious vomiting for 6 days, not passing flatus and stools for 3 days. The patient complained of acute spasmodic pain mainly in the left hypochondrium and lumbar region. There was no significant medical history at the time of admission. There was history of an operative procedure done 30 years back, the details of which were not available.

On admission the patient was severely dehydrated with a pulse rate of $110 / \mathrm{min}$ and a blood pressure of $80 / 60 \mathrm{~mm}$ of $\mathrm{Hg}$. On inspection there was a midline scar in the supraumbilical region. Abdominal examination revealed tenderness in the left hypochondrium and lumbar region. A vague mass was palpable in the left upper quadrant. Bowel sounds were absent. On per-rectal examination the rectum was empty with ballooning with no evidence of fresh bleeding or malena. Investigations revealed hemoglobin of $19.2 \mathrm{mg} / \mathrm{dl}$, WBC count of $19,000 /$ cumm and Serum Creatinine of $2.11 \mathrm{mg} / \mathrm{dl}$. Computed tomography of the abdomen showed an approximately $3.5 \mathrm{cms}$ wide opening along the greater curvature of stomach in the antro-pyloric region through which there was herniation of 
multiple small bowel loops of the ileum along with mesentric vessels. (FIG. 1). However, endoscopy was not performed pre-opertaively.

An exploratory laparotomy was subsequently performed on the patient after giving preoperative antibiotics. Intra-operative findings revealed that the patient had been operated for gastrojejunostomy (retro-colic and iso-peristaltic). The stomach was grossly dilated. The efferent jejunal loop of the jejuno-gastric anastamosis had undergone intussusception in a retrograde manner into the stomach through the stoma site.

The herniated segment of the jejunum could not be reduced and hence a gastrotomy was performed. (FIG. 2) Approximately $10-15 \mathrm{cms}$ of the gangrenous bowel loops were resected. The healthy stump of the efferent segment was anastomosed with the distal jejunum by hand sewn method. The resultant efferent jejunal loop was fixed to the transverse colon to prevent recurrence. In the post-operative period there was no evidence of anastamotic leakage.

The histopathology report of the operated specimen revealed it as a gangrenous small bowel segment. In the post-operative period the patient remained on ventilatory and inotropic support. Subsequently patient succumbed on the fourth post-operative day as a result of septicemia and acute renal failure.

DISCUSSION: Intussusception is an underappreciated complication of Gastrojejunostomy. In one review of 15, 553 gastric bypass surgeries at a single institution, 23 patients developed a retrograde intussusception.[6] There is no medical treatment for jejuno-gastric intussusception and surgical intervention is required for definite treatment. Gastrojejunostomy intussusception can be classified into three anatomical types: afferent loop intussusception; efferent loop intussusception or that involving both afferent and efferent loops.[4]

Two forms of jejuno-gastric intussusception have been clinically recognized: an acute and a chronic form. In the acute form, incarceration and strangulation of the intussuscepted loop generally occurs, whilst spontaneous reduction is usual in the chronic type. The acute form is characterized by sudden severe colicky epigastric pain, vomiting and subsequently, hematemesis. Epigastric tenderness and a palpable abdominal mass can be observed in about $50 \%$ of cases and signs of small bowel intestinal obstruction can also be found. ${ }^{[6,7,8]}$ In the chronic form, the diagnosis is difficult. The main reason for this is that upper GI endoscopy must be performed during the symptomatic period for the diagnosis to be confirmed.[9]

The etiology of Jejuno-gastric intussusception is still not clear. The most likely hypothesis is disordered motility with functional hyper peristalsis triggered by spasm or hyperacidity. Mechanical factors include adhesions, long mesentery and a sudden increase in abdominal pressure. Intussusception of gastro-jejunostomy is more often retrograde. The classical triad of jejuno-gastric intussusception includes sudden onset of epigastric pain, vomiting with or with-out hematemesis and a palpable epigastric mass with past history of gastric surgery.[11] Although computed tomography is the most reliable investigation, endoscopy performed by someone familiar with this rare entity, is certainly diagnostic.[10,11]

Treatment of jejuno-gastric intussusception involves prompt surgery. If the intussuscepted bowel loop is viable and reducible, then simple reduction is performed. To prevent recurrence the reduced jejunal loop can be fixed either to the afferent loop or the transverse colon. If the bowel loop is not viable it should be resected and the continuity with the distal segment should be established. 


\section{REFERENCES:}

1. Bozzi E. Annotation, Bull Acad Med. 1914; 122:3-4.

2. Kshirsagar AY, Shinde SL, Langade YB, Pujari SG, Jeur AS. Retrograde jejunogastric intussusception with strangulation after posterior gastrojejunostomy. MJAFI 2008;64: 282-283.

3. Hasan M, Mahamud MM, Khan SA, Rahman M. Jejunogastric intussusception. Mymensingh Med J. 2009; 18 (2):255-9.

4. ShackmanR. Jejunogastric intussusception. Br. J Surg. 1940; 27: 475-480.

5. Sibley WL. Chronic intermittent intussusception through the stoma of a previous gastroenterostomy. Proc Staff Meet Mayo Clin. 1934; 9 : 364-365.

6. Waits JO, Beart RW Jr, Charboneau JW: Jejunogastric intussusception. Arch Surg 1980; 115: 1449-1452.

7. White TT, Harrison RC, Reoperative Gastrointestinal surgery. Little Brown and Company, 1973. P. 98.

8. Foster Dg, Retrograde Jejunogastric Intussusception - a rare cause of hematemesis. AMA Arch Surg. 1956; 73: 1009-1017.

9. Jaaskelainen V. Retrograde intussusception after certain gastric operation. Ann Chir Gynaecol. 1954; 5 (Suppl):129-135.

10. Kallen R, GraffnerH, Jonsson Per-Ebbe J. Jejunogastric Intususception through the enteroanastomosis after gastric resection. Case report. ActaChir Scand. 1986; 152: 637-640.

11. Truong SN, Tittel A, Schumpelick V. Die jejunogastrische Invagination-eineseltene Komplikation der Magenchirurgie. Z Gastrenterol. 1992; 30:798-800.

Figure 1: Computed tomography showing the site of retrograde intussusception of the jejunal loops into the stomach.

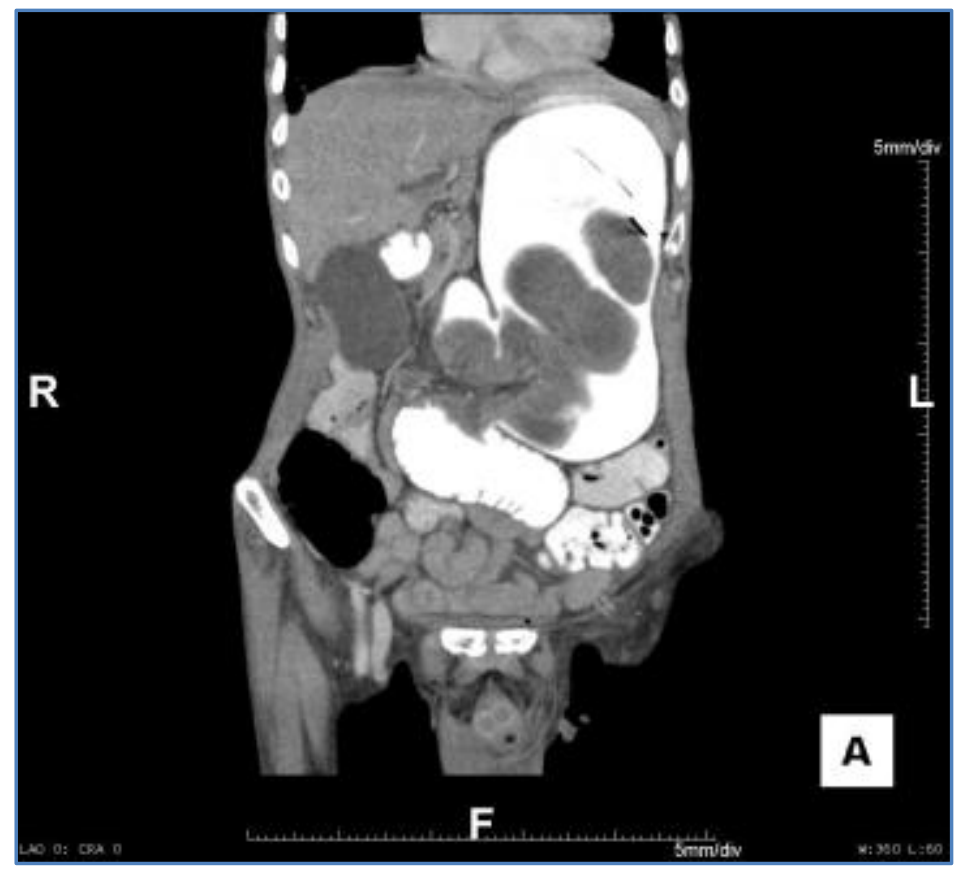

Figure 1 


\section{CASE REPORT}

Figure 2: Gangrenous bowel loops of the efferent segment revealed after gastrotomy.

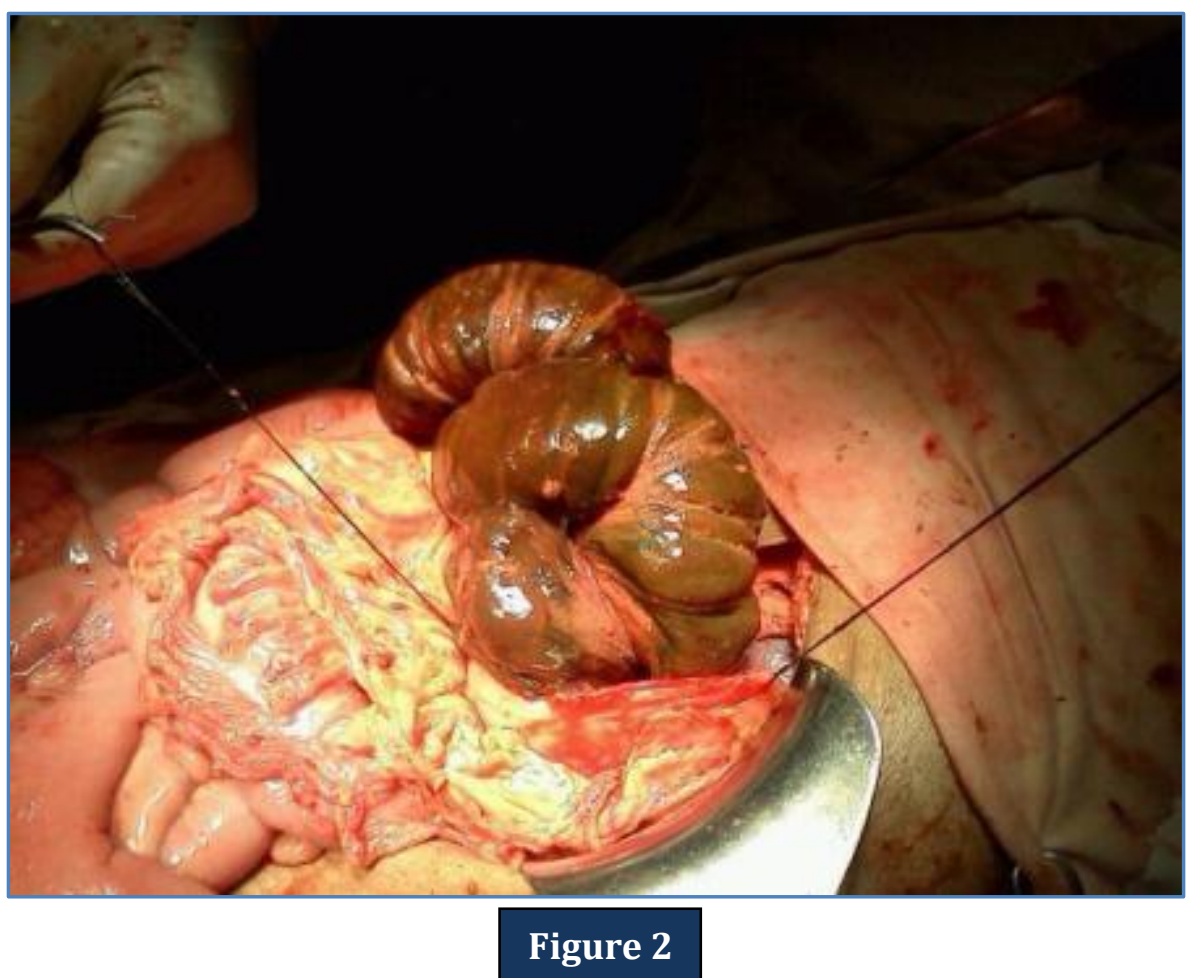

\section{AUTHORS:}

1. Bharat R. Saxena

2. Rajesh C. Mahey

3. Rajesh G. Patil

4. Parag L. Sonawane

\section{PARTICULARS OF CONTRIBUTORS:}

1. Post Graduate Student, Department of General Surgery, TN Medical College and BYL Medical College, Mumbai.

2. Associate Professor, Department of General Surgery, TN Medical College and BYL Nair Hospital, Mumbai.

3. Assistant Professor, Department of General Surgery, TN Medical College and BYL Nair Hospital, Mumbai.

4. Post Graduate Student, Department of General Surgery, TN Medical College and BYL Nair Hospital, Mumbai.

\section{NAME ADDRESS EMAIL ID OF THE CORRESPONDING AUTHOR:}

Dr. Bharat R. Saxena, \#B-301, Ivy Towers, Vasant Valley, Ge A. K. Vaidya Marg, Malad (East), Mumbai-400097.

Email: bharat_sxn@yahoo.co.in

Date of Submission: 18/04/2014.

Date of Peer Review: 19/04/2014.

Date of Acceptance: 20/05/2014.

Date of Publishing: 02/06/2014. 